\title{
Characteristics of hepatic solitary necrotic nodules on contrast-enhanced ultrasonography
}

\author{
Chunyu Lu, Shaoshan Tang ${ }^{*}$, Xiaoyue Zhang, Yang Wang, Kaiming Wang and Peng Shen
}

\begin{abstract}
Background: To summarize the characteristics of solitary necrotic nodules (SNN) in the liver observed under contrast-enhanced ultrasonography (CEUS).

Methods: Conventional ultrasonography (US) and CEUS were performed in 24 patients who were confirmed to have SNN by pathological assessment. The US data and dynamic enhancement patterns of CEUS were recorded and retrospectively analyzed.

Results: Ten of 24 patients underwent surgical resection, while the other 14 patients underwent a puncture biopsy to be confirmed as SNN. Among the 24 patients, 13 patients had a single lesion and 11 patients had multiple lesions. The largest lesion was selected for CEUS examination for patients with multiple lesions. Eleven patients presented no enhancement in all three phases, while the other 13 patients presented with a peripheral thin rim-like enhancement in the arterial phase, an iso-enhancement in the portal phase and delayed phase. However, no enhancement in the interior of the lesions was detected during three phases of CEUS.
\end{abstract}

Conclusions: SNN has characteristic findings on the CEUS, which play an important role in the differential diagnoses of liver focal lesions.

Keywords: Solitary necrotic nodules of liver, Ultrasonography, Contrast agent, Diagnosis, Differentiation, Contrast enhanced ultrasound

\section{Background}

Solitary necrotic nodules (SNN) of the liver are rare non-neoplastic nodular lesions which was first reported by Shepherd in 1983[1]. Until now, the pathogenesis of SNN was not clear, with several theories in the literature as follows [2, 3]: (1) central necrosis of hepatic hemangioma after sclerosis; (2) formation and progression of certain benign lesions after trauma in the liver; (3) parasitic infection; (4) malignant tumor of the digestive tract at the same time, which may lead to coagulative necrosis of liver due to allergic reaction. The clinical symptoms are not obvious, and it is difficult accurately diagnose

\footnotetext{
${ }^{*}$ Correspondence: 824009246@qq.com

Department of Ultrasound, Shengjing Hospital of China Medical

University, 36 Sanhao St, Heping District, Shenyang 110004, Liaoning

Province, China
}

SNN through conventional ultrasound (US) [4]. Most patients undergo enhanced computed tomography (CT) or enhanced magnetic resonance imaging (MRI) for diagnosis. With the wide application of contrast-enhanced ultrasonography (CEUS) in the diagnosis of liver lesions, some hepatic lesions could be qualitatively diagnosed by the enhancement pattern observed under CEUS [5]. This could be helpful to improve the differential diagnosis of hepatic lesions by comparing the difference in blood perfusion between the lesion and its surrounding normal liver parenchyma. In addition, CEUS has the advantage of displaying the lesion's micro-vessels, which is helpful for the preoperative diagnosis of SNN, and avoids unnecessary puncture biopsy or surgical trauma. Since few studies have identified features of SNN on the CEUS for a differential diagnosis. In this study, we aimed to 
summarize the characteristics of hepatic SNN on CEUS and improve the diagnostic accuracy.

\section{Methods \\ Patients}

The ethics committee of our hospital approved this retrospective study and informed consent was obtained from all patients. From December 2010 to May 2020, 24 patients diagnosed with SNN by pathological results (surgical resection or puncture biopsy) in our hospital were analyzed retrospectively. This study included 6 males and 18 females, aged 24 to 67 years old, with an average of $50.33 \pm 11.90$ years. Most of the patients had no obvious clinical symptoms. Fifteen cases were examined by physical examination or hospitalized for other extrahepatic diseases, 2 patients were complicated with abdominal distension (abdominal pain or emaciation because of their gallbladder adenomyosis and gastritis), and 7 patients had a history of malignant tumor ( 2 cases of breast cancer, 3 cases of colon cancer and 2 cases of hepatocellular carcinoma). No patients had undergone any therapy such as interventional ablation or medicinal treatment before CEUS. All patients had Child-Pugh score A, 6 patients had fatty liver, 1 patient had hepatitis $B$ with alpha fetal protein (AFP) elevation, 1 patient had hepatitis $\mathrm{B}$ alone, 3 patients had elevated carcinoembryonic antigen (CEA), and the remaining patients had no history of hepatitis, AFP, CEA, carbohydrate antigen 19-9 and 12-5 levels were normal (Table 1).

\section{Examination acquisition}

US and CEUS examinations were performed using SuperSonic Imagine (Aixplorer ultrasound system, France) or Toshiba Aplio500 (Toshiba Medical Systems, Tokyo, Japan), the central frequency of probe was $3.5-5 \mathrm{MHz}$,

\section{Table 1 General information of the patients [case (\%)]}

\begin{tabular}{ll}
\hline Project & Result \\
\hline Gender & \\
Male & $6(25.00)$ \\
Female & $18(75.00)$ \\
Examination reason & \\
History of malignant & $7(29.17)$ \\
Clinical symptoms & $2(8.33)$ \\
Physical examination & $15(62.50)$ \\
Disease background & \\
Fatty liver & $6(25.00)$ \\
Hepatitis/cirrhosis & $2(8.33)$ \\
Tumor biomarkers increase & \\
AFP & $1(4.17)$ \\
CEA & $3(12.50)$ \\
\hline
\end{tabular}

and the CEUS mechanical index was 0.06-0.15. The ultrasound contrast agent was Sonovue (diameter $2.5 \mu \mathrm{m}$ produced by Bracco Company of Italy), and the main component was sulfur hexafluoride $\left(\mathrm{SF}_{6}\right)$.

\section{US and CEUS}

Conventional US was performed for all patients. Data including number, location, size, boundary, shape, internal echo of liver lesions and color Doppler images were recorded. CEUS was performed by injecting $2.4 \mathrm{ml}$ of contrast agent through the anterior cubital vein, followed by $5 \mathrm{ml}$ of saline solution. The dynamic perfusion process of the contrast agent and enhancement pattern of the lesions were observed and recorded on a hard disk for at least $5 \mathrm{~min}$. The CEUS images were analyzed independently by two doctors (with 15 and 5 years of experience in CEUS imaging).

\section{Statistical analysis}

All statistical analyses were performed with the SPSS version 19.0 software package (SPSS Inc, Chicago, IL), and the quantitative data which were normally distributed are expressed as mean \pm standard deviation $(\bar{x} \pm s)$. The differences in the lesion size between the different enhancement patterns on the CEUS were compared by an independent-samples $t$ test. The differences in the lesion shape between the different enhancement patterns on the CEUS were compared by a Fisher's exact test. A $P$ value less than 0.05 was considered statistically significant.

\section{Results}

\section{Patient diagnosis and outcomes}

All the patients were confirmed as SNN by pathological results, with 10 patients undergoing surgical resection and 14 patients undergoing a puncture biopsy. In the 14 patients with biopsy, the lesions were significantly reduced in 5 patients, and no significant changes were observed in the other 9 patients. One patient relapsed 4 years after surgical resection.

\section{Conventional ultrasound examination}

Among the 24 patients, 13 patients had a single lesion and 11 patients had multiple lesions (six patients with 2 lesions, 4 patients with 3 lesions, 1 patient with 4 lesions). There were 41 lesions in total. The largest lesion was selected for CEUS examination. Among the observed 24 lesions, 19 lesions were located in the right lobe while 5 lesions were located in the left lobe of the liver. Eleven cases had lesions close to the hepatic capsule (the distance between the edge of the lesion and the hepatic capsule was less than $1 \mathrm{~cm}$ ). The maximum diameter of the lesions was $2.41 \pm 0.88 \mathrm{~cm}(1.1-4.1 \mathrm{~cm})$. The boundary of the lesions was clear in 19 cases and unclear in 5 cases. 
The lesions were regularly- round or oval-shaped in 16 cases, claw-like in 2 cases, bead-like in 1 case, dumbbelllike in 3 cases and nodular-like in 2 cases. The lesions were hypo-echoic in 18 cases, hyper-echoic in 2 cases, and heterogeneous mixed in 4 cases. There was no obvious blood flow signal in all lesions, and normal hepatic blood vessels could be seen around the lesions in 16 cases.

\section{Contrast-enhanced ultrasonography}

The CEUS enhancement pattern was divided into 2 types. Type I was no enhancement in all three phases compared with the surrounding liver parenchyma (Fig. 1): 11 cases belonged to type I. Type II was presence of peripheral thin rim-like hyper-enhancement in the arterial phase and iso-enhancement in the portal phase and delayed phases, with no enhancement in the interior of the lesions during all three phases (Figs. 2, 3, 4): 13 cases showed a type II enhancement pattern. The nonenhanced area remained unchanged during three phases (Table 2). The boundary of the enhancement ring was clear and sharp. The thickness of the enhancement ring was $0.30 \pm 0.15 \mathrm{~cm}$. There was a significant difference in lesion size between the two enhancement patterns $(P=0.005<0.05)$. The lesions of type II $(2.83 \pm 0.87 \mathrm{~cm})$ were larger than lesions of type I $(1.92 \pm 0.61 \mathrm{~cm})$. There was no significant difference in lesion shape between the two enhancement patterns $(P=0.211>0.05)$.

\section{Pathology}

The resected section of the specimen was yellow or white, and some of them were gray-white or grayish yellow, with or without a thin capsule, and the boundary was clear. The textures of the lesions were slightly hard and partially fused. Hematoxylin and eosin (HE) staining showed that the lesions were mainly homogeneous coagulation necrotic tissue, surrounded by proliferated fibrous tissue, with surrounding focal infiltration of inflammatory cells and multinucleated giant cells, and punctate necrosis surrounding the liver tissue.

\section{Discussion}

$\mathrm{SNN}$ is a rare non-neoplastic lesion of the liver which often occurs in patients aged between 60 to 70 years, but it had also been reported to occur between the ages of 30 to 40 years, with more males being affected than females [6]. Most patients showed no clinical symptoms, and were detected occasionally during physical examination, postmortem examination, or operation [7]. The pathological features of SNN include nodular coagulation necrosis, and presence of a complete fibrous capsule around the lesion, with the infiltration of inflammatory cells in the capsule (mainly eosinophil cells and lymphocytes), and no vascular tissue in the lesion $[4,8]$.

It had been reported that the $\mathrm{SNN}$ lesions could reduce or disappear after conservative treatment. So, if SNN is highly suspected, surgical resection should be avoided [9, 10]. Therefore, a correct diagnosis before the operation is crucial.

There are no specific clinical symptoms and laboratory tests for the diagnosis of SNN $[4,11]$. SNN often presents as hypo-echoic nodules on the conventional US, which lacks specificity, and thus makes it difficult to make a qualitative diagnosis. [12-14]. Color Doppler US has some limitations in the detection of the micro-vessels in the lesions, and puncture biopsy or surgical resection are the ultimate diagnostic methods and gold standard for SNN detection [15]. With the development of CEUS, the process of internal and peripheral blood perfusion can be dynamically observed in real time, which could effectively improve the accuracy of SNN diagnosis and reduce unnecessary biopsy or surgical trauma [16, 17].

In this study, 11 patients $(11 / 24)$ showed no enhancement in the three phases, which was similar to the report by Wang et al.[18]. However, in our study, 13 patients $(13 / 24)$ showed peripheral thin rim-like enhancement in the arterial phase, which could easily be misdiagnosed as a malignant tumor such as metastatic hepatic carcinoma (MHC). However, the inner wall of the lesion's enhancement ring was clear and sharp, and demonstrated isoenhancement in the portal and delayed phases, which differed from the obviously decreased enhancement ring of the MHC lesion in the portal phase [2, 8]. Peripheral thin rim-like enhancement in the arterial phase suggested micro-vessel distribution around the lesions of the SNN. HE staining showed that the edges of the lesions were hyperplastic fibrous tissue and inflammatory response bands caused by infiltrated inflammatory cells. This might be the pathological basis of the peripheral thin rim-like enhancement observed in the arterial phase [4]. Some lesions showed septum-like enhancement, which might be related to fibrous tissue separation following

\footnotetext{
(See figure on next page.)

Fig. 1 Images in a 60-70-year-old sex "2". a There was a hypo-echoic lesion under the hepatic capsule in segment 3 of the liver with a size of $2.2 \mathrm{~cm} \times 2.1 \mathrm{~cm}$. No obvious blood flow signal was detected in the lesion. $\mathbf{b}$ No contrast agent filling was found in the arterial phase (timer, 00: 24), portal phase (timer, 01:47), and delayed phase (timer, 02: 26). There was no enhancement in all the three phases. $\mathbf{c}$ Enhanced CT showed no obvious enhancement in the lesions. $\mathbf{d}$ The resected section of the specimen showed that the lesion was yellow-white and the boundary was clear. A large area of necrotic tissue with surrounding focal inflammatory cell infiltration could be seen (hematoxylin and eosin, $\times 100$ )
} 

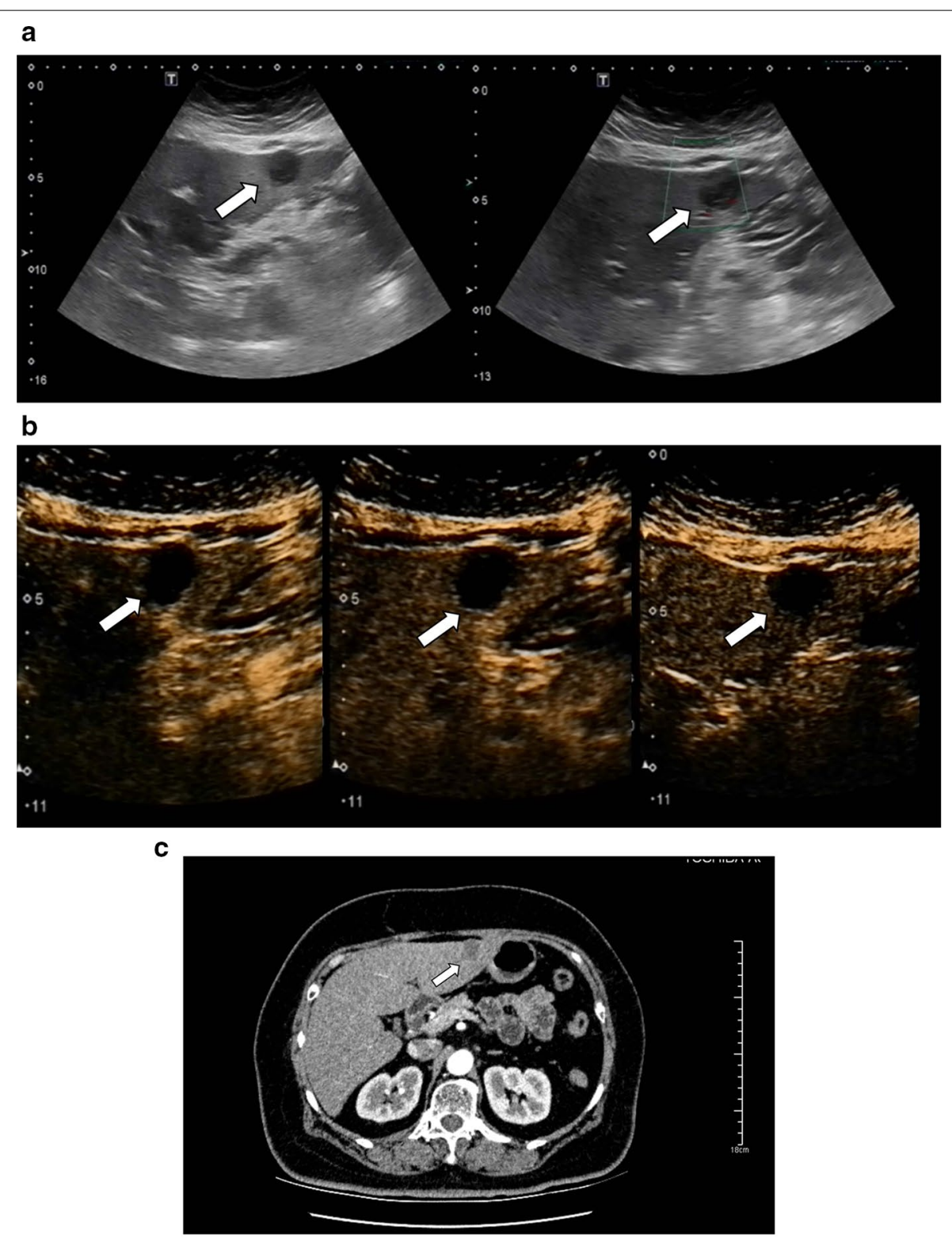

d

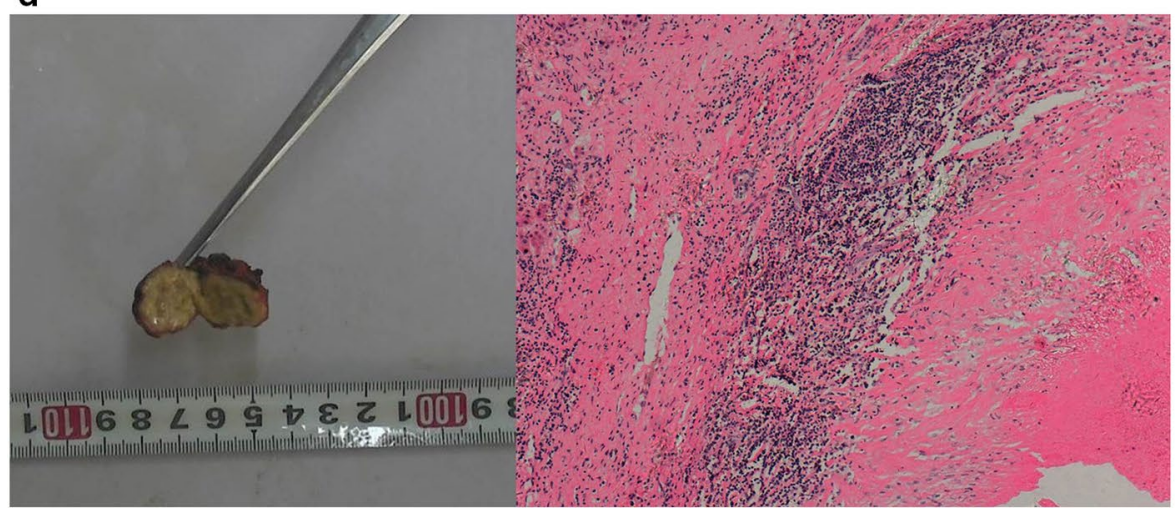



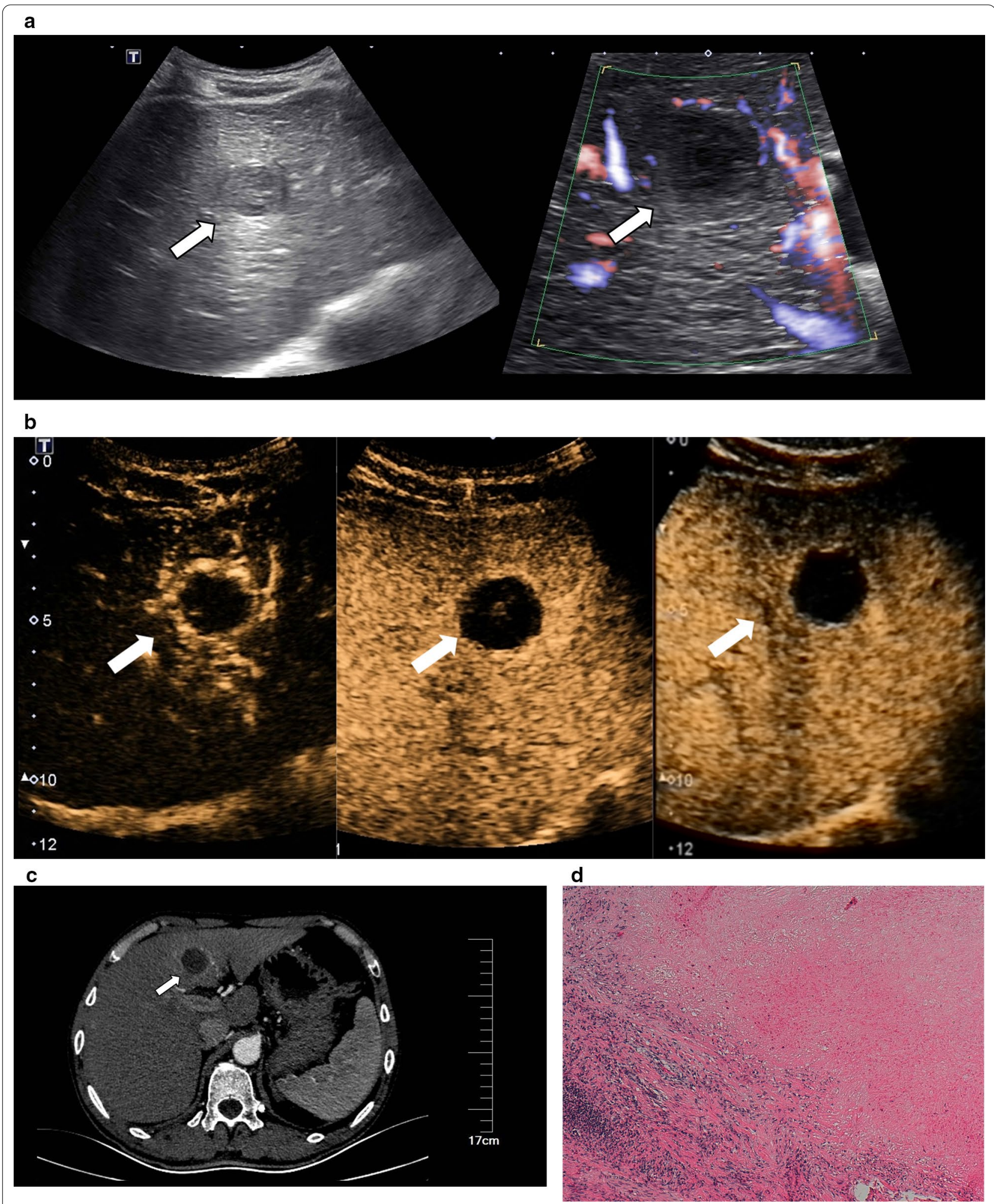
(See figure on previous page.)

Fig. 2 Images in a 50-60-year-old sex "1" with a history of hepatocellular carcinoma 3 years after surgery. a There was a hypo-echoic lesion in segment 4 of the liver with a size of $3.0 \mathrm{~cm} \times 2.5 \mathrm{~cm}$. Normal hepatic blood vessels could be seen around the lesions but no obvious blood flow signal was detected. $\mathbf{b}$ In arterial phase (timer, 00: 13), the lesions showed obvious thin peripheral rim-like enhancement, and the thickness of the enhanced ring was $0.36 \mathrm{~cm}$; this demonstrated iso-enhancement in the portal phase (timer, 01: 29) and delayed phase (timer, 04:10). There was no contrast agent filling in the interior of the lesions. $\mathbf{c}$ Enhanced computed tomography showed no obvious enhancement in the lesions but enhancement in the boundary of the lesion. $\mathbf{d}$ Necrotic tissue of the surrounding liver tissue and focal inflammatory cell infiltration could be seen (hematoxylin and eosin, $\times 100$ )

multi-lesion fusion. Internal septum-like enhancement may be one of the characteristics of multi-lesion fusion. There was a significant difference in lesion size between the two enhancement patterns: the lesions which showed a peripheral thin rim-like enhancement were larger than those without enhancement. This suggested that the lesions were in different pathologic stage: those without enhancement might be at the end-stage and tended to atrophy. The lesions with peripheral thin rim-like enhancement might be at the relatively early stage and maintain the peripheral vessel distribution.

Previous literature has shown that presence of a marked peripheral rim-like enhancement with internal hypo-intensity on a longer MRI (a delayed time of 1-2 h) was helpful in the diagnosis of SNN [19]. The contrast agent used during an MRI is different from that used during a CEUS. The ultrasonic contrast agent is a blood-pool imaging agent, which does not diffuse to the intercellular space and does not show delayed enhancement. The MRI contrast agent is an extracellular contrast agent, which can diffuse to the extracellular space. Because the contrast medium diffuses slowly between blood vessels and the fibrous tissue, the peripheral rim-like enhancement of the lesions is observed 1-2 $\mathrm{h}$ after injection of the contrast agent. The interval is relatively long, making it unsuitable for routine clinical examination. SNN shows specific manifestations at the early stage of CEUS, which could reduce the examination time and gave better repeatability, thus making CEUS more suitable for clinical use.

SNN with peripheral rim-like enhancement in the arterial phase should especially be distinguished from MHC. Previous studies had reported that when MHC lesions appeared to be necrotic, it could present peripheral rim-like enhancement in the arterial phase with the interior of the lesion showing no enhancement in the three phases [20-22]. However, there was a significant difference in the thickness of the enhancement ring in the arterial phase between MHC and SNN. The enhancement rings of the MHC were thicker than that of the SNN [23]. Besides, the enhancement ring of the MHC was infiltrated by malignant tissue, and the contrast agent was washed out during the portal phase and demonstrated hypo-enhancement. Therefore, the hypoechoic area became larger in the delayed phase. However, the enhancement ring of the SNN was a thin fibrous capsule without obviously being washed out in the delayed phase; therefore, the hypoechoic area showed no significant change compared the arterial phase [24].

The peripheral rim-like enhancement observed on the CEUS can also be detected in some cases of intrahepatic cholangiocarcinoma (ICC). However, the enhancement ring in ICC is unevenly thicker with an irregular shape, and the interior of the lesion has a contrast agent filling. Clinical characteristics and specific laboratory examinations are also helpful for the differential diagnosis of SNN [25-27].

When an abscess with liquefied and necrotic tissue is formed, a peripheral rim-like enhancement is observed in the arterial phase of CEUS, but honeycomb enhancement of the internal part can help for differentiation [28-30].

Hepatic alveolar echinococcosis (HAE) is also a rare parasitic disease with a long incubation period and lack of specific clinical symptoms [31]. There is no contrast media filling in lesions during all the three phases in HAE. The combination of contact history in the epidemic area and specific laboratory examination could be helpful for a differential diagnosis [32, 33].

SNN needs to be differentiated from hypovascular hepatocellular carcinoma (HCC). Although more than

\footnotetext{
(See figure on next page.)

Fig. 3 Images in a 40-50-year-old sex "2". a There were two hypo-echoic lesions in segment 7 of the liver which connected like a dumbbell. Normal hepatic blood vessels could be seen inside (the lesions connected portion) and around the lesions. $\mathbf{b}$ In the arterial phase (timer, 00:24), the lesions showed thin peripheral rim-like enhancement, and the thickness of the enhanced ring was $0.23 \mathrm{~cm}$; this showed iso-enhancement in the portal phase (timer, 00: 57) and delayed phase (timer, 02: 03). There was no contrast agent filling in the interior of the lesions. $\mathbf{c}$ Enhanced CT showed no obvious enhancement in the lesions but showed enhanced blood vessels. $\mathbf{d}$ A large area of necrotic tissue with surrounding inflammatory cell infiltration could be seen (hematoxylin and eosin, $\times 100$ )
} 

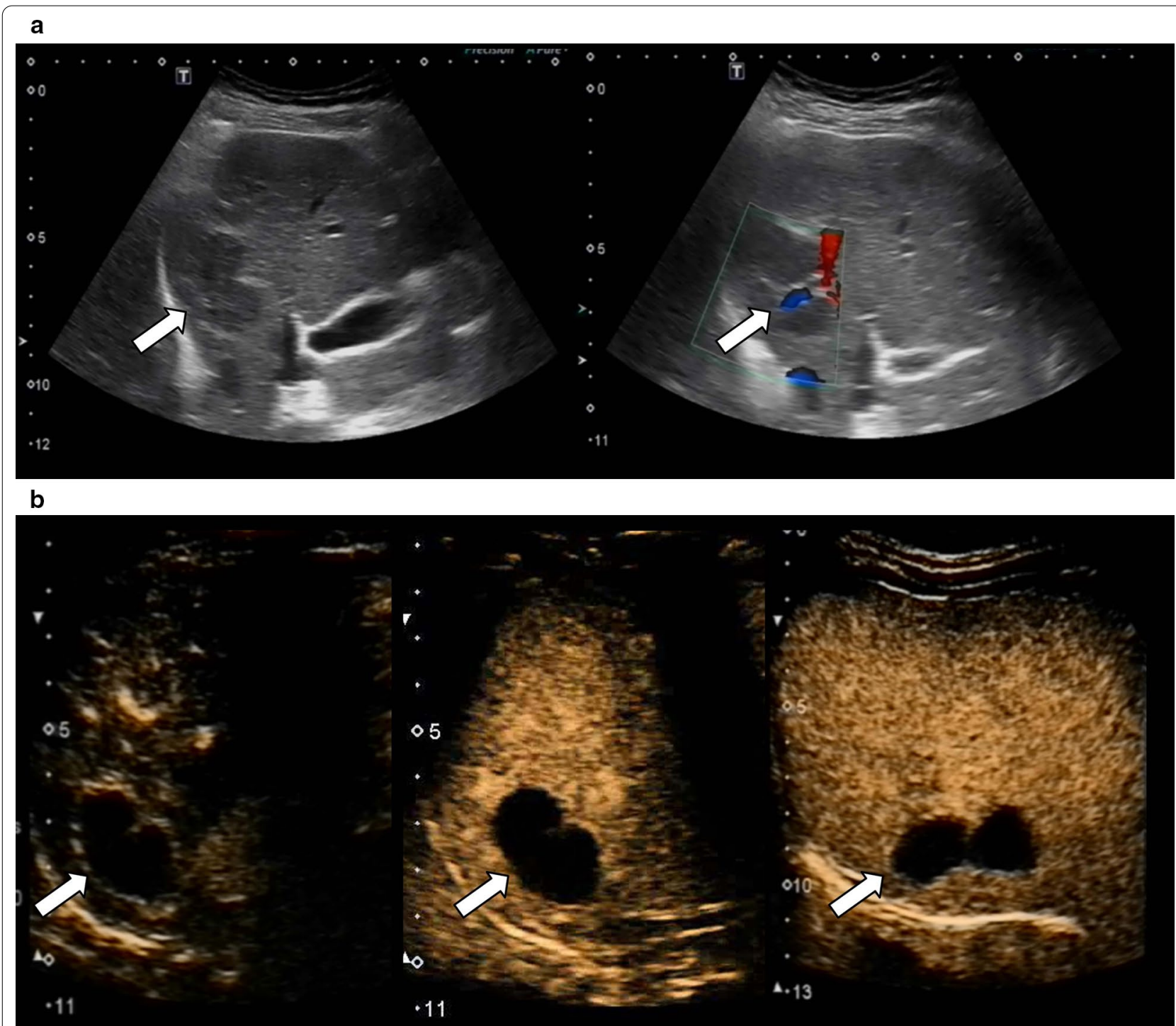

c

d
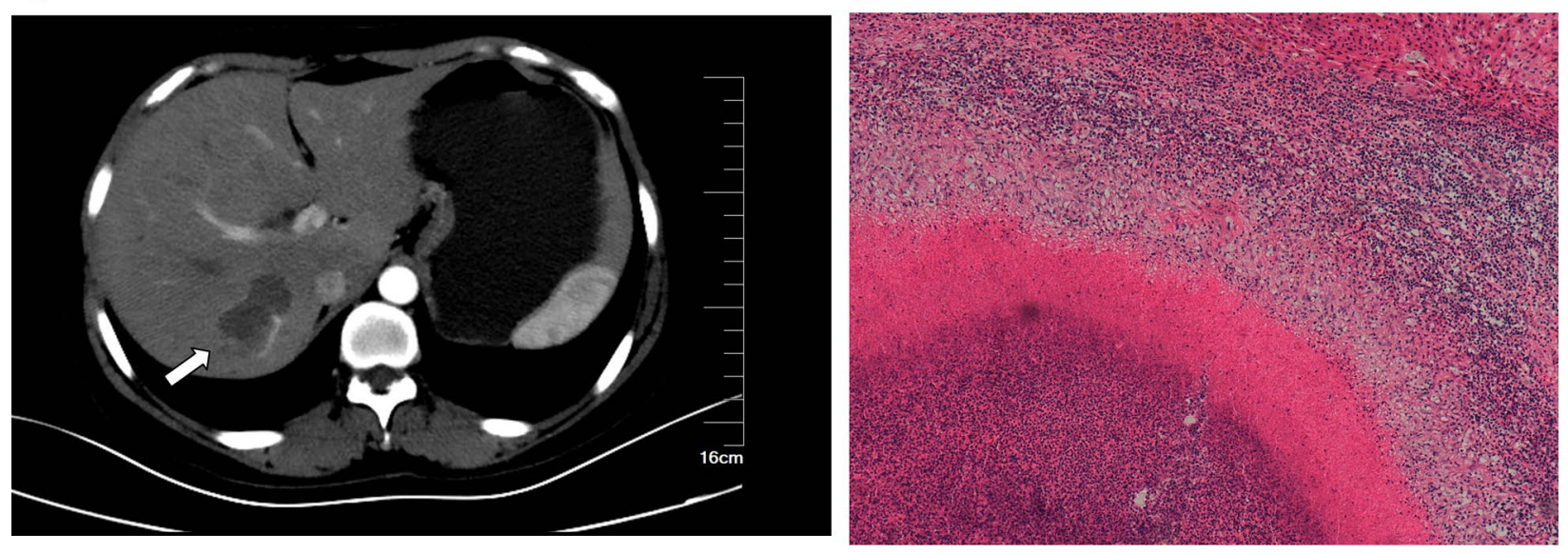


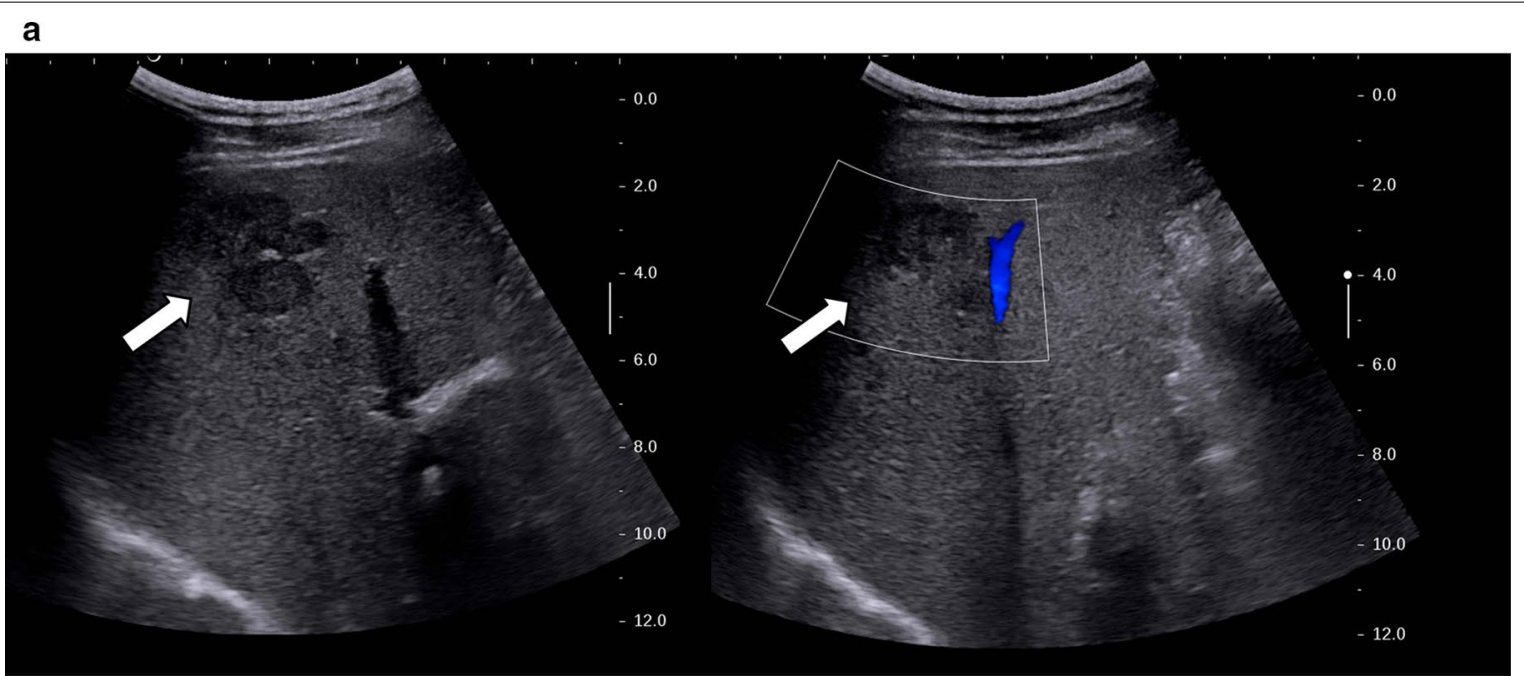

\section{b}

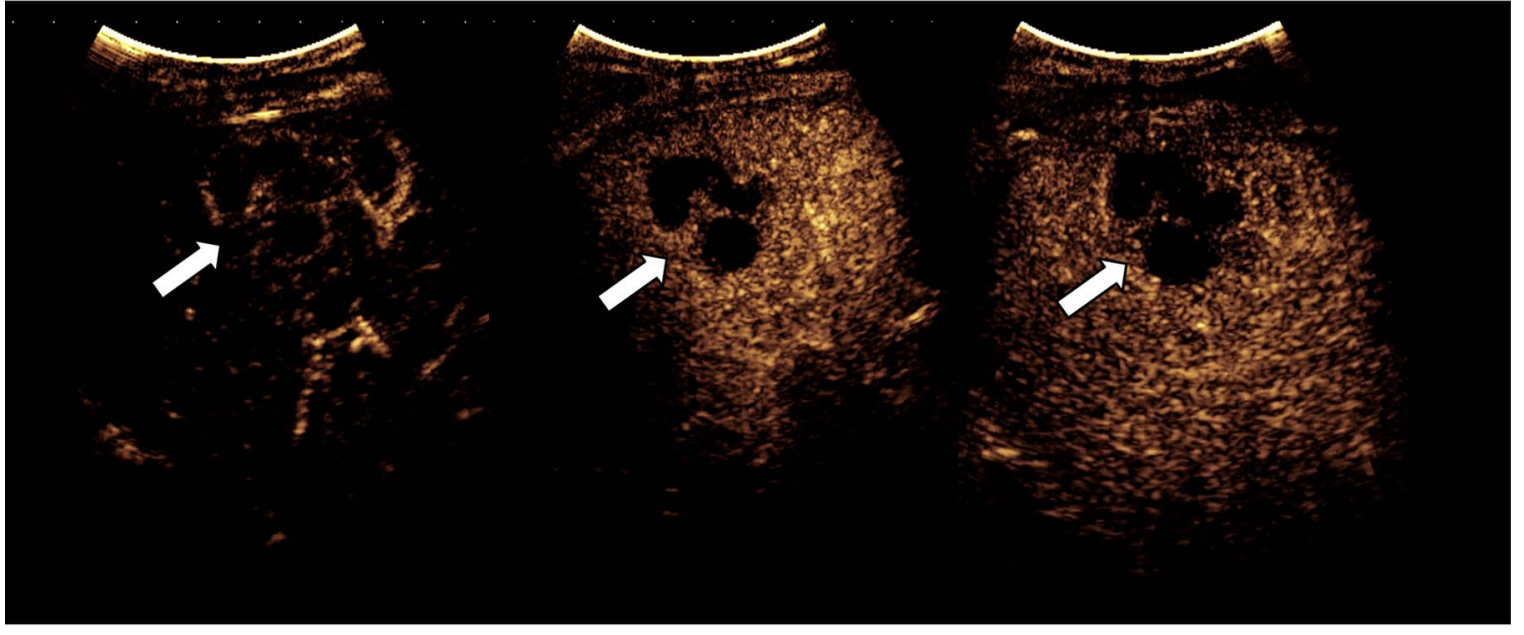

c

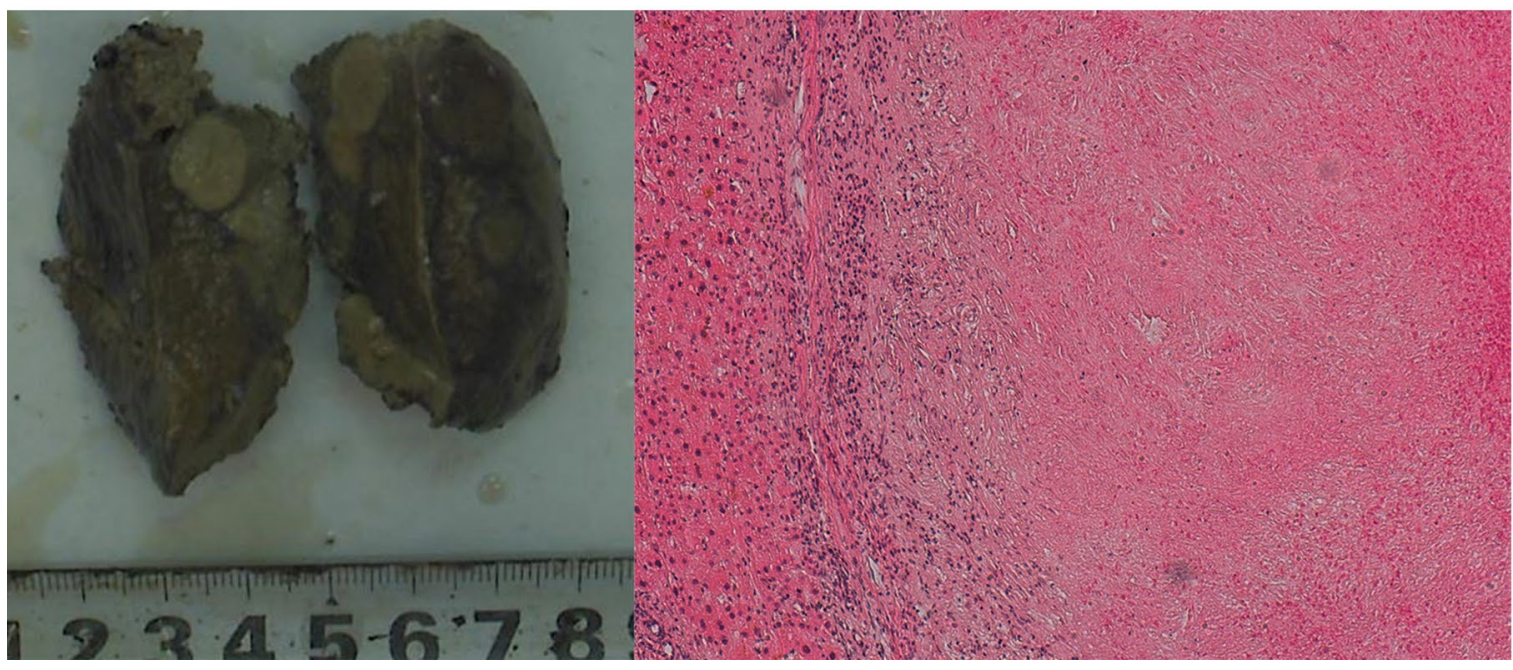


(See figure on previous page.)

Fig. 4 Images in a 50-60-year-old sex "1". a. Several hypo-echoic lesions were fused in segment 5 of the liver, which presented as a claw-like shape, with a size of approximately $3.8 \mathrm{~cm} \times 2.8 \mathrm{~cm}$. Color Doppler did not detect obvious blood flow signal in the lesions, and normal hepatic blood vessels could be seen in the surrounding region. b. In arterial phase (timer, 00:20), the lesions showed thin peripheral rim-like enhancement and septum enhancement in the interior lesions, but most of the lesions were not enhanced, and the thickness of the enhanced ring around the lesions was $0.21-0.25 \mathrm{~cm}$. The enhanced portion of the lesions showed iso-enhancement in the portal phase (timer, 00:40) and delayed phase (timer, 02:05), and the center of the lesions were not enhanced in these two phases. c. On the section of the resected specimen, the lesions were yellow white, with focal lesions fusing, and the boundary was clear. The lesions were necrotic tissue, and hyperplastic fibrous tissue and inflammatory cell were seen around the lesions(hematoxylin and eosin, $\times 100$ )

Table 2. 24 patients of SNN US and CEUS imaging [case (\%)]

\begin{tabular}{ll}
\hline Project & Result \\
\hline Single/multiple lesions & \\
Single lesion & $13(54.17)$ \\
Multiple lesions & $11(45.83)$ \\
Lesion site & \\
Left lobe & $5(20.83)$ \\
Right lobe & $19(79.17)$ \\
Distance between lesion and hepatic capsule & \\
$>1$ cm & $13(54.17)$ \\
$<1$ cm & $11(45.83)$ \\
Lesion morphology & \\
Regularity & $16(66.67)$ \\
Claw-like & $2(8.33)$ \\
Bead-like & $1(4.17)$ \\
Nodular-like & $2(8.33)$ \\
Dumbbell-like & $3(12.50)$ \\
Echo oflesion & \\
Hypo-echoic & $18(75.00)$ \\
Hyper-echoic & $2(8.33)$ \\
Heterogeneous echoic & $4(16.67)$ \\
Lesion boundary & \\
Clear & $19(79.17)$ \\
Unclear & $5(20.83)$ \\
Normal hepatic blood vessels in the periphery & \\
Yes & $16(66.67)$ \\
No & $8(33.33)$ \\
Enhancement pattern & $11(45.83)$ \\
No enhancement & $13(54.17)$ \\
\hline & \\
thin rim-like enhancement & \\
\hline
\end{tabular}

$90 \%$ of the HCC are hypervascular, few of HCC may showing little or no obvious enhancement in the inner of the lesion on CEUS, but there is no obvious rim enhancement around the lesion. It is difficult to differentiate HCC from SNN to those lesions without obvious enhancement. Puncture biopsy can be performed when it is difficult to differentiate HCC from SNN. Hepatitis or liver cirrhosis background and the increase of AFP are also helpful to the differential diagnosis [34, 35].
One case of this study relapsed 4 years after surgery, which has not been previously reported. The lesions showed no enhancement in all three phases during both CEUS examinations between four years. Therefore, although SNN is a benign lesion, there is a possibility of recurrence. Such patients who choose conservative treatment require attention and should be closely followed up.

This study was the first to report two types of enhancement patterns observed in SNN, and a case of relapse, which has not been reported in the literature. However, some limitations should also be noted, as this study was a retrospective study and with a relatively small number of cases. A larger sample size and prospective study is required for further investigation.

\section{Conclusions}

In conclusion, SNN has specific characteristic findings on the CEUS, which can improve the diagnostic accuracy and CEUS can be an effective technique for the diagnosis of SNN.

\section{Abbreviations}

AFP: Alpha fetal protein; CEA: Carcinoembryonic antigen; CEUS: Contrastenhanced ultrasonography; CT: Computed tomography; HAE: Hepatic alveolar echinococcosis; HCC: Hepatocellular carcinoma; HE: Hematoxylin and eosin; ICC: Intrahepatic cholangiocarcinoma; IPL: Inflammatory pseudo-tumor of the liver; MHC: Metastatic hepatic carcinoma,; MRI: Magnetic resonance imaging, SNN: Solitary necrotic nodules; US: Conventional ultrasound.

\section{Acknowledgements}

We thank all the editors and anonymous reviewers for their helpful suggestions on improving the quality of our article.

\section{Authors' contributions}

Authors made substantial contributions to the conception OR design of the work (CL, ST); OR the acquisition (CL, ST, XZ, YW, KM), analysis (CL, ST, PS) OR interpretation of data; OR have drafted the work or substantively revised it (all authors) AND approved the submitted version (and any substantially modified version that involves the author's contribution to the study) (all authors); AND agreed both to be personally accountable for the author's own contributions and to ensure that questions related to the accuracy or integrity of any part of the work, even ones in which the author was not personally involved, are appropriately investigated, resolved, and the resolution documented in the literature (all authors). All authors have read and approved the manuscript in its current state.

\section{Funding}

Not applicable. 


\section{Availability of data and materials}

The raw data generated and analyzed in the current study are not publicly available due to appropriate protection of patient personal information but are available from the corresponding author on reasonable request.

\section{Ethics approval and consent to participate}

The study was approved by the ethics committee of Shengjing Hospital of China Medical University (2017PS155J). All procedures followed were in accordance with the ethical standards of the responsible committee on human experimentation (institutional and national) and with the Helsinki Declaration of 1975, as revised in 2008. This study is a retrospective analysis, it did not include any human trial, and all participants provided written informed consent.

\section{Consent for publication}

Not applicable.

\section{Competing interests}

The authors declare that they have no competing interests.

Received: 2 July 2020 Accepted: 12 January 2021

Published online: 25 January 2021

\section{References}

1. Shepherd NA, Lee G. Solitary necrotic nodules of the liver simulating hepatic metastases. J Clin Pathol. 1983;36(10):1181-3.

2. Zhou YM, Li B, Xu F, et al. Clinical features of solitary necrotic nodule of the liver. Hepatobiliary Pancreat Dis Int. 2008;7(5):485-9.

3. Deniz K, Coban G. Solitary necrotic nodule of the liver: Always benign? J Gastrointest Surg. 2010;14(3):536-40

4. Pananwala $H$, Pang TC, Eckstein RP, et al. The enigma of solitary necrotic nodule of the liver. ANZ J Surg. 2014;84(4):260-5.

5. Piscaglia F, Nolsøe C, Dietrich C, et al. The EFSUMB guidelines and recommendations on the clinical practice of contrast enhanced ultrasound (CEUS): Update 2011 on non-hepatic applications. Ultraschall Med. 2012:33(01):33-59.

6. Delis SG, Kelgiorgi DA, Sofianidis AA, et al. Solitary necrotic nodule of the liver mimicking hepatocellular carcinoma: a case report. Cases J. 2009;2(1):85 .

7. 7Zimmermann A. Solitary Necrotic Nodule of the Liver. Tumors and Tumor-Like Lesions of the Hepatobiliary Tract. 2016; 2183-9.

8. Molins IG, Font JMF, Ivaro JC, et al. Contrast-enhanced ultrasound in diagnosis and characterization of focal hepatic lesions. World J Radiol. 2010;2(12):455-62.

9. Martins RJT, Tralhão JG, Cipriano MA, et al. Solitary necrotic nodule of the liver: a very challenging diagnosis. BMJ Case Reports. 2014. https://doi. org/10.1136/bcr-2013-202364.

10. Choi CS, Cho EY, Jeong JS, et al. Spontaneous regression of a solitary necrotic nodule of the liver. Hepatol Int. 2010;4(3):649-52.

11. Noh SJ, Jachin S, Moon WS. Solitary necrotic nodule of the liver. Clin Mol Hepatol. 2012;18(2):235-8.

12. Han XJ, Sun CK, Li CM. Ultrasonographic findings of solitary necrotic nodule of liver. Chin J Med Imaging Technol. 2003;19(9):1171-2.

13. Xing H, Wang DQ, Xu Y, et al. Analysis of contrast-enhanced ultrasonography in diagnosis of hemodynamics of intrahepatic solitary necrotic nodule. J Mod Med Health. 2014;30(19):2900-1.

14. Ni XX, Zhou HY, Wei XD. Contrast-enhanced ultrasonography of solitary necrotic liver nodule. Chin J Med Ultrasound. 2011:8(7):1451-6.

15. Imura S, Miyake K, Ikemoto T, et al. Rapid-growing solitary necrotic nodule of the liver. J Med Invest. 2006;53(3-4):325-9.

16. Lu Y, Liu B, Zheng Y, et al. Application of real-time three-dimensional contrast-enhanced ultrasound using SonoVue for the evaluation of focal liver lesions: a prospective single-center study. Am J Transl Res. 2018;10(5):1469-80
17. Dietrich CF, Ignee $A$, Greis C, et al. Artifacts and pitfalls in contrastenhanced ultrasound of the liver. Ultraschall Med. 2014;35(2):108-28.

18. Wang $Y, Y u X$, Tang J, et al. Solitary necrotic nodule of the liver: contrastenhanced sonography. J Clin Ultrasound. 2007;35(4):177-81.

19. Fang JY, Ma XY, Yu DX, et al. Specific imaging characteristic of solitary necrotic nodule of the liver: Marked peripheral rim-like enhancement with internal hypointensity on longer delayed MRI. Eur Radiol. 2017;27(9):1-11.

20. Tatsuo I, Masatoshi K, Osamu M, et al. Value of liver parenchymal phase contrast-enhanced sonography to diagnose premalignant and borderline lesions and overt hepatocellular carcinoma. AJR. 2009;192(3):698-705.

21. Otani K, Chijiiwa K, Kondo K, et al. Enlarged solitary necrotic nodule of the liver misinterpreted as a metastatic liver cancer. Clin J Gastroenterol. 2009;2(5):355-60.

22. Lu Q, Zhang XL, Han H, et al. Value of perfusion parameters for differentiating hepatocellular carcinoma and liver metastasis with hypervascularity and a normal hepatic background on contrast-enhanced ultrasound imaging. J Ultrasound Med. 2019;38(10):2601-8.

23. Li J, Tang SS, Yu HW. Value of contrast-enhanced ultrasound in differential diagnosis of single metastatic liver cancer and solitary necrotic nodule of the liver. J Clin Hepatol. 2016;32(7):1338-41.

24. Yue WW, Wang S, Xu HX, et al. Parametric imaging with contrastenhanced ultrasound for differentiating hepatocellular carcinoma from metastatic liver cancer. Clin Hemorheol Microcirc. 2016;64(2):177-88.

25. Rimola J, Forner AM. Cholangiocarcinoma in cirrhosis: absence of contrast washout in delayed phases by magnetic resonance imaging avoids misdiagnosis of hepatocellular carcinoma. Hepatology. 2010:50(3):791-8.

26. Chen TJ, Chang XY, Lv K, et al. Contrast-enhanced ultrasound features of intrahepatic cholangiocarcinoma: a new perspective. Sci Rep. 2019;9(1):19363.

27. Tian SY, Xu D, Wang YJ, Yu YH, Yang Y, Jiang TA. Diagnostic value of contrast-enhanced ultrasonography for intrahepatic cholangiocarcinoma with tumor diameter larger than $5 \mathrm{~cm}$. Hepatobiliary Pancreat Dis Int. 2020. https://doi.org/10.21203/rs.3.rs-44537/v2.

28. Sun K, Zhu W, Luo Y, Li Y, Zhou X. Transient segmental enhancement of pyogenic liver abscess: a comparison between contrast-enhanced ultrasound and computed tomography. J Comput Assist Tomogr. 2018:42(1):133-8.

29. Popescu A, Sporea I, Sirli R, et al. Does contrast enhanced ultrasound improve the management of liver abscesses? A single centre experience. Med Ultrason. 2015;17(4):451-5.

30. Kishina M, Koda M, Tokunaga S, et al. Usefulness of contrast-enhanced ultrasound with Sonazoid for evaluating liver abscess in comparison with conventional B-mode ultrasound. Hepatol Res. 2015;45(3):337-42.

31. Cai DM, Li YZ, Jiang Y, Wang HY, Wang XL, Song B. The role of contrastenhanced ultrasound in the diagnosis of hepatic alveolar echinococcosis. Medicine. 2019;98(5):e14325.

32. Cai DM, Wang HY, Wang $X \mathrm{~L}$, et al. Ultrasonographic findings of small lesion of hepatic alveolar echinococcosis. Acta Trop. 2017:174:165-70.

33. Kaltenbach TEM, Graeter T, Mason RA, et al. Determination of vitality if liver lesions by alveolar echinococcosis Comparison of parametric contrast enhanced ultrasound (SonoVue (R)) with quantified F-18-FDGPET-CT. Ultraschall Med. 2015:54(1):43-9.

34. Hayashi $\mathrm{H}$, Nishigaki $Y$, Tomita $\mathrm{E}$, et al. Usefulness of early vascular phase images from contrast-enhanced ultrasonography using Sonazoid for the diagnosis of hypovascular hepatocellular carcinoma. Hepatol Res. 2016;46(6):497-504

35. Wang JH, Lu SN, Hung $\mathrm{CH}$, et al. Small hepatic nodules $(\leq 2 \mathrm{~cm})$ in cirrhosis patients: Characterization with contrast-enhanced ultrasonography. Liver Int. 2006:26(8):928-34.

\section{Publisher's Note}

Springer Nature remains neutral with regard to jurisdictional claims in published maps and institutional affiliations. 